\title{
Ueber Phenyldihalogenpropionsăuren,
} insbesondere uber Phenylchlorjodpropionsäure und einige Derivate derselben;

von Emil Erlenmeyer.

Die von den Phenyldihalogenpropionsäuren zuerst bekannte Phenyldibrompropionsäure ist fast gleichzeitig von Ad. Schmidt ${ }^{1}$ ) und von $\operatorname{mir}^{2}$ ) durch Einwirkung von Bromdampf auf Zimmtsäure erhalten worden. Später hat sie Glaser ${ }^{3}$ ) in der gleichen Weise dargestellt, aber er bekam sie dann auch noch ${ }^{4}$ ) durch Einwirkung von Bromwasserstoff auf Phenylmilchsäure. Darauf haben Fittig und Binder ) die Methode der Addition von Brom an Zimmtsäure in der Weise abgeändert, dass sie zu einer Lösung von Zimmtsäure in wasserfreiem Schwefelkohlenstoff die berechnete Menge Brom gleichfalls in Schwefelkohlenstoff gelöst hinzubrachten. Da aber 1 Theil Zimmtsäure nach unserer Bestimmung ${ }^{6}$ ) bei $15^{0} 109,6$ Theile Schwefelkoblenstoff zur Lösung erfordert, so haben wir für die Darstellung grösserer Mengen des Additionsproductes gleiche Theile feingepulverte Zimmtsäure und Schwefelkohlenstoff mit der berechneten Menge Brom, in der dreifachen Gewichtsmenge Schwefelkohlenstoff gelöst, allmählich vermischt, öfter umgeschüttelt und unter Abktuhlung 24 Stunden in Berührung gelassen. Die nach dem Abgiessen des Schwefelkohlenstoffs bleibende schmierige Masse wurde auf Thontellern getrocknet und ergab als Rohproduct analysirt 51,46 statt $51,94 \mathrm{pC}$. Brom.

Phenyldichlorpropionsäure versuchte zuerst Glaser ${ }^{7}$ ) durch Einwirkung rauchender Salzsäure auf Phenylchlormilchsäure

1) Diese Annalen 126, 254 und 127, 319.

9) Ztschr. f. Chemie 6, 307 und 7, 545.

s) Diese Amnalen 148, 331.

4) Diese Annalen 147, 91.

5) Diese Annalen 195, 140.

e) Inaug-Dissert. von H. Stockmeier, Nürnberg 1883, 26.

7) Diese Annalen 147, 91.

Anzalen der Chemie 289. Bd. 
darzustellen, es gelang ihm aber nicht, ein Product in der zur Analyse erforderlichen Reinheit zu gewinnen. In Gemeinschaft mit G. W. Jutz habe ich dann die Phenyldichlorpropionsäure ${ }^{8}$ ) zunächst durch Einleiten von Chlor in eine Schwefelkohlenstoffrösung von Zimmtsäure dargestellt. Wir bekamen dieselbe aber auch vollständig rein, in Krystallform und allen übrigen Eigenschaften mit dem Additionsproduct ubereinstimmend, durch Einwirkung von Chlorwasserstoff auf Phenylchlormilchsäure.

Phenyldijodpropionsäure habe ich vergeblich darzustellen versucht; sowohl in ätherischer als in Schwefelkohlenstofflösung blieben Jod und Zimmtsäure auch nach tagelanger Berührung und dann folgendem allmählichen Verdunsten des Lösungsmittels unverbunden (vgl. auch Li ebermann, Ber. d. deutsch. chem. Ges. 24, 4113).

Phenylchlorbrompropionsäure hat Glas e ${ }^{9}$ ) einerseits durch Einwirkung von Bromwasserstoff auf Phenylchlormilchsäure, andererseits von Chlorwasserstoff auf Phenylbrommilchsäure gewonnen. Er sagt darüber 1. c. 92: „Die nach beiden Methoden erhaltenen Säuren sind nach vollkommener Reinigung nicht von einander zu unterscheiden; sie zeigen vollkommene Uebereinstimmung in ihren physikalischen Eigenschaften und ich bin daher genöthigt, sie als identisch anzunehmen." Nach der Anschauung, welche Glaser damals tiber die Constitution der Zimmtsäure hatte, war diese Annahme auch ganz gerechtfertigt und naturlich.

Glaser hielt für die Constitution der Zimmtsäure die Formel :

$$
\mathrm{C}_{6} \mathrm{H}_{5}-\mathrm{CH}_{2}-\mathrm{C}-\mathrm{COOH}
$$

für richtiger, als die von mir aufgestellte:

$$
\mathrm{C}_{6} \mathrm{H}_{5}-\mathrm{CH}=\mathrm{CH}-\mathrm{COOH} \text {. }
$$

9) Ber. d. deutsch. chem. Ges. 14, 1867 und Jutz, Inaug.-Dissert. München 1882; vergl. auch Li ebermann, Ber. d. deutsch. chem. Ges. 28, 2235.

9) Diese Annalen 147, 92-94. 
Demzufolge musste sowohl die Phenylchlormilchsäure, als auch die Phenylbrommilchsäure Halogen und Hydroxyl an dem $\alpha$-Kohlenstoff enthalten:

$\mathrm{C}_{6} \mathrm{H}_{5}-\mathrm{CH}_{2}-\mathrm{COH} . \mathrm{Cl}-\mathrm{COOH} ; \mathrm{C}_{6} \mathrm{H}_{5}-\mathrm{CH}_{2}-\mathrm{COH} \cdot \mathrm{Br}-\mathrm{COOH}$

und wenn das Hydroxyl in der ersten durch Brom, in der zweiten durch Chlor ersetzt wurde, so musste in beiden Fällen Phenyl- $\alpha$-chlor- $\alpha$-brompropionsäure entstehen.

Obgleich sich Glas er später ${ }^{10}$ ) gezwungen sah, zur Annahme meiner Constitutionsformel der Zimmtsäure überzugehen, so hat er sich doch nicht darüber ausgesprochen, welche Constitution danach den Phenylhalogenmilchsäuren zukomme, auch die Frage, ob die beiden Phenylchlorbrompropionsäuren identisch oder isomer seien, blieb noch eine offene, nachdem ich für die beiden Phenylhalogenmilchsäuren die Constitution:

$$
\mathrm{C}_{6} \mathrm{H}_{5} \text { - } \mathrm{CHOH}-\mathrm{CHX}-\mathrm{COOH} \text {, }
$$

dass sie also Phenylhalogenhydracrylsäuren seien, nachgewiesen batte; denn es konnte ja als möglich angenommen werden, dass die beiden Halogenatome an den beiden Kohlenstoffatomen sich so ordneten, dass in jedem Falle das Brom die $\alpha$ - und das Chlor die $\beta$-Stellung (oder umgekebrt) einnehme.

Es erschien daher nicht als überflüssig, die beiden Dihalogensäuren aus Phenylbrommilchsäure mit Chlorwasserstoff und aus Phenylchlormilchsäure mit Bromwasserstoff einer erneuten Untersuchung zu unterwerfen.

Beide Säuren wurden nach dem von Glaser angegebenen Verfahren dargestellt ${ }^{11}$ ). Es zeigte sich nun, dass die beiden Säuren nach der von Herrn Professor Haus h ofer ausgeführten krystallographischen Untersuchung miteinander, sowie mit Phenyldibrom - und Phenyldichlorpropionsäure isomorph sind, dagegen wurde der Schmelzpunkt der Säure aus Phenylbrommilchsäure und Chlorwasserstoff bei $182^{\circ}$, derjenige aus Phenylchlormilchsäure und Bromwasserstoff bei $184,5^{\circ}$ liegend gefunden. Ihre

\footnotetext{
10) Diese Annalen 154, 138.

11) Inaug-Dissert. Stockmeier, 34 ff.
} 
Constitutionsisomerie ergab sich jedoch unzweideutig, als die beiden Säuren in gleicher Weise der Destillation mit Wasserdaupf unterworfen wurden.

Die Säure aus Phenylbrommilchsäure und Chlorwasserstoff erwies sich als Phenyl- $\beta$-chlor- $\alpha$-brompropionsäure, denn sie lieferte Phenylbrommilchsäure und $\alpha$-Bromstyrol, die andere wurde als Phenyl- $\beta$-brom- $\alpha$-chlorpropionsäure erkannt, denn sie lieferte Phenylchlormilchsäure und $\alpha$-Chlorstyrol. Die durch Halogenwasserstoff eingeführten Halogenatome waren also in beiden Fällen an die Stelle des Hydroxyls getreten und hatten je ihren Platz behauptet. Damit ist nun entschieden, dass die beiden Säuren nicht identisch, sondern in der angegebenen Weise isomer sind.

Es waren bisher also die folgenden Dihalogenüre der Zimmtsäure (Phenyldihalogenpropionsäuren) bekannt:

$\mathrm{C}_{8} \mathrm{H}_{5}$ - $\mathrm{CHBr}-\mathrm{CHBr}-\mathrm{COOH} ; \mathrm{C}_{6} \mathrm{H}_{5} \mathrm{CHCl}-\mathrm{CHCl}-\mathrm{COOH}$;

$\mathrm{C}_{6} \mathrm{H}_{5}$-CHCl-CHBr-COOH; $\mathrm{C}_{6} \mathrm{H}_{5}-\mathrm{CHBr}-\mathrm{CHCl}-\mathrm{COOH}$.

Nun handelte es sich noch darum, ob es nicht möglich sei, ein gemischtes Dihalogenür der Zimmtsäure, in welchem das eine Halogen Chlor, das andere Jod wäre d. h. Phenylchlorjodpropionsäure darzustellen. Wenn auch, wie Glas er gefunden hat, Jodwasserstoff auf Phenylchlor- wie auf Phenylbrommilchsäure unter Abscheidung von freiem Jod einwirkt, also jedenfalls in dieser Weise das gewünschte Dihalogenür der Zimmtsäure nicht zu erwarten war, und obgleich, wie ich und auch Liebermann gefunden hatte, die Jodatome in den Jodmolekülen so fest verbunden sind, dass sie durch die Zimmtsäure nicht getrennt und addirt werden können, so war es immer noch denkbar, dass in den Chlorjodmolekülen die beiden Halogenatome weniger fest gebunden seien und sich mit der Zimmtsäure vereinigen liessen. Von ganz besonderem Interesse war dabei noch die Frage, welches der beiden Halogene die $\alpha$ - und welches die $\beta$-Stellung einnehme. Nach den Erfahrungen, welche man uber die Stellungnahme von Wasserstoff und Halogen bei der Bildung der Halogenwasserstoffadditionsproducte der 
Zimmtsäure gemacht hatte, liess sich wohl voraussetzen, dass das negativere der beiden Halogene, das Chlor, die $\beta$ - und das weniger negative Jod die $\alpha$-Stellung besetzen würde.

Da die Darstellung von reinem Einfach-Chlorjod einige Schwierigkeiten bietet, habe ich vorgezogen, die von Schützenberger ${ }^{12}$ ) entdeckte, leicht $\mathrm{zu}$ gewinnende und beständige Chlorjodsalzsäure $(\mathrm{JCl}+\mathrm{ClH})$ zu benutzen. Man erhält dieselbe, wenn Jod in Salzsäure vertheilt und dann unter Umschütteln allmählich Natriumnitrit eingetragen wird, bis alles Jod in Lösung gegangen ist. Aus der angewendeten Menge Jod lässt sich der Gehalt an Chlorjodsalzsäure in dem ganzen Flüssigkeitsvolum resp. in $1 \mathrm{ccm}$ berechnen.

Bei den zuerst unternommenen Versuchen, das Chlorjod aus der wässrigen Flüssigkeit an die Zimmtsăure anzulagern, wurde nicht Phenylchlorjodpropionsäure, sondern Phenylhydroxyjodpropionsäure und zwar, wie unten gezeigt werden wird, Phenyl- $\beta$-hydroxy - $\alpha$-jodpropionsäure (Phenyljodhydracrylsäure) erhalten.

Hiernach unterliegt es kaum einem Zweifel, dass in der ersten Phase der Einwirkung von Chlorjod auf die Zimmtsäure zwar ein Chlorjodadditionsproduct entstanden ist, dass sich dieses aber in der wässrigen Flüssigkeit unter Abspaltung von Chlorwasserstoff in $\beta$-Lacton verwandelte, welches dann durch Aufnahme von Wasser in die Phenyljodhydracrylsäure überging, wie es folgende Formeln versinnlichen:

I. $\mathrm{C}_{8} \mathrm{H}_{5}-\mathrm{CH}=\mathrm{CH}-\mathrm{COOH}+\mathrm{ClJ}=\mathrm{C}_{6} \mathrm{H}_{5}-\mathrm{CHCl}-\mathrm{CHJ}-\mathrm{COOH}$.

II. $\mathrm{C}_{6} \mathrm{H}_{5}$-CHCl-CHJ- $\mathrm{COOH}-\mathrm{HCl}=\mathrm{C}_{6} \mathrm{H}_{5}$ - $\mathrm{CH}$-CHJ<smiles></smiles>

III. $\mathrm{C}_{6} \mathrm{H}_{5}-\mathrm{CH}-\mathrm{CHJ}+\mathrm{HOH}=\mathrm{C}_{0} \mathrm{H}_{5}$ -

Die Bildung der Phenyljodhydracrylsăure aus der zuerst entstandenen Phenylchlorjodpropionsäure liesse sich freilich auch

12) Jahresber. 187\%, 216. 
noch so annehmen, dass direct an die Stelle des Chlors Hydroxyl eingetreten wäre:

$\mathrm{C}_{6} \mathrm{H}_{5}$ - $\mathrm{CHCl}-\mathrm{CHJ}-\mathrm{COOH}+\mathrm{HOH}=\mathrm{C}_{6} \mathrm{H}_{5}$ - $\mathrm{CHOH}-\mathrm{CHJ}-\mathrm{COOH}+\mathrm{HCl}$

Ich halte aber die erste Annahme für wahrscheinlicher, weil, wie sich gezeigt hat, die Ester der Zimmtsäure in der gleichen wässrigen Lösung der Chlorjodsalzsäure nicht in Phenyljodhydracrylsäureester, sondern in Phenylchlorjodpropionsäureester verwandelt werden. Es lässt sich hieraus schliessen, dass das Wasser nicht im Stande ist, direct Hydroxyl an die Stelle des Chlors einzuführen, sondern dass die Bildung der Hydroxysäure nur durch Hydrolyse des $\beta$-Lactons möglich ist. Die Lactonbildung kann aber hier nicht stattfinden, weil das in den Estern an der Stelle des Wasserstoffs des Carboxyls befindliche Alkyl nicht mit dem Chlor in der $\beta$-Stellung als Alkylchlorür auszutreten vermag.

Es musste nun noch versucht werden, ob nicht doch auch die freie Phenylchlorjodpropionsäure durch Addition von Chlorjod an Zimmtsäure erhalten werden könne, wenn von vornherein das Wasser von der Reaction ganz ausgeschlossen würde. Da sich nach $\mathrm{Schatzenberger}$ die Chlorjodsalzsäure der wässrigen Lösung durch Aether unverändert entziehen lässt, so wurde eine solche ätherische Lösung mit trockner Zimmtsäure zusammengebracht. Und so gelang es in der That, wie unten näher beschrieben wird, die freie Phenylchlorjodpropionsäure zu gewinnen.

Diese wird aber, wie sich nach dem oben Mitgetheilten erwarten liess, durch kaltes Wasser nach einiger Zeit, durch Wasser von $70^{\circ}$ augenblicklich vollständig in Phenyljodhydracrylsäure verwandelt. Dieselbe Umwandlung tritt augenblicklich ein, wenn man ein Mol.-Gew. Kaliumbydroxyd in wässriger Lösung auf ein Mol.-Gew. Phenylchlorjodpropionsäure einwirken lässt.

Eine, wie es mir scheint, analoge Veränderung erleidet die Phenylchlorjodpropionsäure, wenn man sie mit einem Mol.Gew. Kaliumhydroxyd in absolut-alkoholischer Lösung behandelt. 
Es bildet sich unter Abscheidung von Chlorkalium Phenyl- $\beta$ äthoxy- resp. -methoxy- $\alpha$-jodpropionsäure.

Da anzunehmen ist, dass hierbei in erster Linie das Kaliumsalz der Phenylchlorjodpropionsäure entsteht, so muss man sich denken, dass weiter durch Vereinigung des Kaliums dieses Salzes mit dem Chlor neben Chlorkalium zunächst $\beta$-Lacton gebildet wird, das sich dann aber durch die Wirkung des Alkohols in die Aethoxysäure verwandelt:<smiles>CCCCC1OOC1C1CCCCC1C=CCO</smiles>

Unter der nicht sehr wahrscheinlichen Voraussetzung, dass nicht in erster Iinie das Kaliumsalz der Phenylchlorjodpropionsäure gebildet wird, liessen sich noch zwei andere Reactionsweisen denken:

$$
\begin{aligned}
& \text { A. } \mathrm{C}_{6} \mathrm{H}_{5}-\mathrm{CHCl}-\mathrm{CHJCOOH}+\mathrm{KOC}_{2} \mathrm{H}_{5}= \\
& \mathrm{KCl}+\mathrm{C}_{6} \mathrm{H}_{6}-\mathrm{CHOC}_{2} \mathrm{H}_{5} \text {-CHJ-COOH. } \\
& \text { B. I. } \mathrm{C}_{6} \mathrm{H}_{5}-\mathrm{CHCl} \text {-CHJ-COOH }+\mathrm{KOO}_{2} \mathrm{H}_{5}= \\
& \mathrm{KCl}+\mathrm{C}_{8} \mathrm{H}_{5} \mathrm{OH}+\mathrm{C}_{6} \mathrm{H}_{5}-\mathrm{CH}=\mathrm{CJ}-\mathrm{COOH} \text {. }
\end{aligned}
$$

B. II. $\mathrm{C}_{6} \mathrm{H}_{5}-\mathrm{CH}=\mathrm{CJ} \cdot \mathrm{COOH}+\mathrm{C}_{2} \mathrm{H}_{5} \mathrm{OH}=\mathrm{C}_{6} \mathrm{H}_{5}-\mathrm{CHOC}_{2} \mathrm{H}_{5}-\mathrm{CHJ}-\mathrm{COOH}$.

Die Reactionsweise $A$ ist mir deshalb nicht sehr wahrscheinlich, weil mir kein Fall bekannt ist, wo ein Halogen in der Beta-Stellung einer Carbonsäure direct durch Aethoxyl substituirt wird.

B. I. wäre ja der regelrechte Verlauf der Reaction von zwei Mol.-Gew. alkoholischem Kali auf ein Mol.-Gew. einer $\alpha \beta$-Dihalogencarbonsäure. B. II, Addition von Alkohol an doppelt gebundene Kohlenstoffatome, ist meines Wissens aber nur einmal bei den Estern der Paranitrophenyl- $\alpha$-nitroacrylsäure von Friedländer ${ }^{13}$ ) beobachtet worden. Welche von den drei angeführten Reactionsweisen die wahrscheinlichste ist, lässt sich schwer entscheiden, zumal da ich es leider versäumt habe, die Nebenproducte, welche die Aethoxysäure begleiten, qualitativ und quantitativ genauer zu verfolgen.

13) Ber. d. deutsch. chem. Ges. 16, 851 . 
Noch muss ich erwähnen, dass auch versucht warde, aus der Phenyljodhydracrylsäure durch einen grösseren Ueberschuss von 38 procentiger Salzsäure Phenylchlorjodpropionsäure zu gewinnen. Es resultirte dabei aber eine Verbindung von der Zusammensetzung $\mathrm{C}_{18} \mathrm{H}_{16} \mathrm{ClJO}_{4}$, welche die Bestandtheile von einem Mol. Phenylchlorjodpropionsäure und einem Mol. Zimmtsäure enthält. Da nach dem unten zu beschreibenden Verhalten dieser Verbindung ihre beiden Componenten nicht durch Kohlenstoffaffinitäten verkettet sein konnten, dachte ich mir früher ${ }^{14}$ ), sie seien in folgender Weise mit einander verbunden:

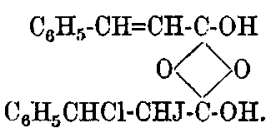

Heute halte ich es aber für wahrscheinlicher, dass sich das Carboxyl der Phenylchlorjodpropionsäure an die Doppelbindung der Kohlenstoffatome der Zimmtsäure in folgender Weise angelagert hat:

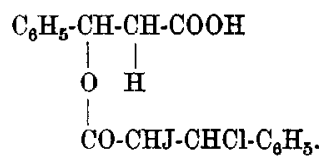

Wir hätten dann eine Verbindung, in welcher der Wasserstoff des Carboxyls der Phenylchlorjodpropionsäure in ähnlicher Weise wie in den Estern durch ein Kohlenwasserstoffradical substituirt wäre und deshalb auch wie bei den Estern das Chlor in seiner Stellung bleiben könnte. Ich denke mir, dass die Phenyljodhydracrylsäure durch die rauchende Salzsäure zunächst in Phenylchlorjodpropionsäure übergeführt wurde, dass dann aber durch die anziehende Wirkung der Salzsäure auf das Chlorjod dieses aus der Hälfte der Moleküle herausgenommen und einerseits Chlorjodsalzsäure, andererseits Zimmtsäure gebildet wurde, welche letztere sich dann mit der zweiten Hälfte der Moleküle der Phenylchlorjodpropionsäure in der angenommenen Weise vereinigte.

14) Ber. d. deutsch. chem. Ges. 10, 2456. 
Wenn man statt der 38 procentigen nur 25 procentige Salzsäure auf die Phenyljodhydracrylsäure einwirken lässt, so werden 98 pC. derselben in Zimmtsäure verwandelt, indem Chlorjodsalzsäure gebildet wird. Es entsteht also auch hier zunächst Phenylchlorjodpropionsäure, dieser wird aber durch die verdünntere Salzsäure das ganze Chlorjod entzogen, während bei Anwendung concentrirter Salzsäure in einem bestimmten Verhältniss sich ein Gleichgewichtszustand in der Anziehung der Zimmtsäure einerseits und der Salzsäure andererseits auf das Chlorjod herstellt. Lässt man grössere Mengen concentrirter Salzsäure einwirken, so wird auch von ihr eine gewisse Menge Zimmtsäure in Freiheit gesetzt.

Bei der Darstellung der Phenyljodhydracrylsäure durch Einwirkung der wässrigen Chlorjodsalzsäurelösung auf Zimmtsäure war es mir auffallend, dass sich dabei nicht auch Jodstyrol bildete, ich habe deshalb die Reactionsbedingungen verschiedentlich abgeändert. Bei allen Versuchen machte sich ein aromatischer Geruch bemerklich und einmal bekam ich auch einige Tropfen einer braunen öligen, aromatisch riechenden Flüssigkeit, deren Jodgehalt annähernd auf den des Jodstyrols stimmte, es gelang mir aber nicht, Styrol daraus darzustellen. Da nun auch alle Versuche, aus Chlor- und aus Bromstyrol durch Behandeln mit Jodkalium in verschiedener Weise Jodstyrol zu erbalten, resultatlos blieben, habe ich es schlieslich aufgegeben, auf Jodstyrol zu fahnden. Die geringe Menge, welche sich anfangs bildet, wird wohl in der wässrigen Flüssigkeit in Phenyläthylaldehyd verwandelt und durch diesen der aromatische Geruch erzeugt.

Die Phenyljodhydracrylsaure bildet wasserfreie Krystalle und solche, welche 1 Molekül Krystallwasser enthalten; durch Natriumamalgam und Wasser wird sie zu Phenylhydracrylsäure reducirt, von Alkalien wird sie in phenylglycidsaures Salz verwandelt. In diesen Punkten verhallt sie sich den beiden anderen Phenylhalogenhydracrylsäuren analog. Bei Reactionen, bei welchen die Jodatome $z \mathfrak{u}$ Jodmolekülen zusammentreten 
können, zeigt die Phenyljodhydracrylsäure ein abweichendes Verhalten. Erhitzt man sie im zugeschmolzenen Rohre mit Wasser im siedenden Wasserbade oder destillirt sie mit Wasserdampf, dann scheidet sich krystallinisches Jod aus, indem sich neben Zimmtsäure Phenyläthylaldehyd und Styrol bildet. $\mathrm{Da} \mathrm{Be}$ stimmungen des Jods und der Zimmtsäure ergaben, dass auf ein Mol.-Gew. freies Jod nahezu ein Mol.-Gew. Zimmtsäure entsteht, so muss man annehmen, dass zwei Moleküle Phenyljodhydracrylsäure bei der Reaction betheiligt sind. Wahrscheinlich wird wie bei den anderen Phenylhalogenhydracrylsäuren aus einem Theil der Säure Phenylglycidsäure resp. Phenyläthylaldehyd und Jodwasserstoff gebildet. Der Jodwasserstoff kann nun auf einen anderen Theil der Phenyljodhydracrylsäure in folgender Weise einwirken:

$\mathrm{C}_{6} \mathrm{H}_{5}$-CHOH-CHJ-COOH $+\mathrm{JH}=\mathrm{J}_{9}+\mathrm{C}_{6} \mathrm{H}_{5}$ - $\mathrm{CHOH}-\mathrm{CH}_{2}-\mathrm{COOH}$.

Die so gebildete Phenylhydracrylsäure wird nun zum grössten Theil in Zimmtsäure und Wasser, zu einem geringen Theil in Styrol und Kohlendioxyd zersetzt.

Gerade weil neben Zimmtsäure auch Styrol entsteht, denke ich mir, dass beiden die Bildung von $\beta$-Lacton vorausgeht. Die Zimmtsäure wird dann in der Weise gebildet, dass die mit $\operatorname{dem} \beta$-Kohlenstoff verbundene Sauerstoffaffinität sich mit einem Wasserstoffatom in der $\alpha$-Stellung vereinigt und so wieder Carboxyl hergestellt wird:

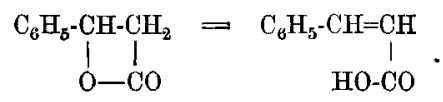

Diese Annahme balte ich für wahrscheinlicher als die, nach welcher das Hydroxyl in der $\beta$-Stellung mit einem Wasserstoffatom in der $\alpha$-Stellung als Wasser austritt,

Das Styrol entsteht aus dem $\beta$-Lacton, indem Kohlendioxyd gedildet wird und $\alpha$ - und $\beta$-Kohlenstoff in doppelte Bindung treten:

$$
\begin{gathered}
\mathrm{C}_{8} \mathrm{H}_{5}-\mathrm{CH}-\mathrm{CH}_{2} \\
\vdots+1 \\
0-\mathrm{CO}
\end{gathered}=\mathrm{C}_{8} \mathrm{H}_{5}-\mathrm{CH}=\mathrm{CH}_{2}+\mathrm{O}=\mathrm{CO} .
$$


Freilichwohl lässt sich die Bildung der Zimmtsäure auch noch in anderer Weise denken. Der in erster Linie entstehende Jodwasserstoff loönnte auch an die Stelle des Hydroxyls der Phenyljodhydracrylsäure Jod einführen, und aus der so gebildeten Phenyldjjodpropionsäure witrden die beiden Jodatome als Molekül austreten, weil wie es scheint ein Dijodür der Zimmtsäure nicht existenzfähig ist. Das Styrol kann aber wohl nur in der oben angenommenen Weise entstehen.

Bei allen zum Zwecke des Studiums der Wirkung des Wassers auf die Phenyljodhydracrylsäure angestellten Versuchen blieb immer eine geringe Menge der Saure unzersetzt, wenn so lange destillirt worden war, bis die Flüssigkeit in der Retorte kein freies Jod mehr enthielt. Es zeigte sich nun das Merkwürdige, dass, wenn man eine wässrige Zimmtsäurelösung mit Jod kocht, bis kein freies Jod mehr vorhanden ist, die Flüssigkeit neben Zimmtsäure Phenyljodhydracrylsäure enthält; denn wenn man sie mit überschüssiger Sodalösung erwärmt und mit verdünnter Schwefelsäure versetzt, so wird Phenyläthylaldehyd frei und die Flussigkeit giebt mit Silbernitrat einen reichlichen Niederschlag von Jodsilber. Man muss hiernach annehmen, dass doch vorubergehend eine geringe Menge Phenyldijodpropionsäure gebildet wird, die sich aber in derselben Weise, wie die anderen Dihalogensäuren in der heissen wässrigen Flüssigkeit gleich weiter verändert.

\section{Experimenteller Theil.}

\section{Phenylchlorjodpropionsäure.}

Zur Darstellung dieser Säure wurde zuerst durch Ausschütteln der wässrigen Lösung der Chlorjodsalzsäure mit Aether eine atherische Lösung dieser Verbindung bereitet. Nachdem dieselbe noch mit Chlorcalcium getrocknet war, wurde darin eine etwas geringere als die molekulare Menge Zimmtsăure aufgelöst. Zunächst war keinerlei Reaction bemerkbar, die I,ösung wurde 
deshalb in einem Becherglase neben Schwefelsäure und Kalihydrat unter eine Glocke gebracht. In dem Maasse als der Aether verdunstete, schieden sich aus der gelben Flüssigkeit farblose Krystalle aus, die sich bei näherer Untersuchung als reine Zimmtsäure erwiesen. Als sich durch weitere Verdunstung des Aethers soviel Krystalle ausgeschieden hatten, dass ein dicker Brei entstand, machte sich zunächst Entwicklung von Chlorwasserstoff bemerkbar. Von nun an wurden die Krystalle durch häufiges Umrühren mit der Flüssigkeit in möglichst innige Berührung gebracht. Es liess sich dann auch eine Veränderung der Krystalle und eine Zunahme der Chlorwasserstoffentwicklung beobachten. Sobald letztere nachgelassen und der Brei eine festere Consistenz angenommen hatte, wurde er auf einer flachen Schale ausgebreitet und bis zum vollständigen Festwerden und Aufhören der Chlorwasserstoffentwicklung unter der Glocke stehen gelassen; die gelbe Farbe batte sich dann in hellrosa verwandelt.

Da sich beim allmählichen Verdunsten des Aethers zunächst noch unveränderte Zimmtsäure abschied und erst Reaction eintrat, nachdem der Aether nahezu vollständig verdunstet war, so wurde bei den weiteren Darstellungen wie folgt verfahren. Von der wăssrigen Chlorjodsalzeäurelösung wurde eine $1^{1 / 8}$ Mol.-Gew. $\mathrm{ClJ}+\mathrm{ClH}$ enthaltende Menge mit Aether extrahirt, die Lösung mit Chlorcalcium getrocknet und soweit destillirt, dass schon gelbe Dämpfe anfingen uberzugehen. In den Rückstand wurde ein Mol.-Gew. Zimmtsäure als feinstes Pulver eingerührt. Schon nach kurzer Zeit beginnt unter Chlorwasserstoffentwicklung Reaction. Man lässt nun unter häufigem sorgfältigem Verreiben der Masse so lange unter der Glocke neben Kalihydrat und Schwefelsäure stehen, bis kein Chlorwasserstoff mehr entweicht.

Wie bei der ersten Darstellungsmethode wird das vollkommen trockne Krystallmehl in trocknem, heissem Chloroform gelöst und die mit trocknem Ligroïn vermischte Lösung erkalten gelassen. Es scheidet sich bald eine reichliche Krystallisation von fast farblosen Blättchen $a b$, welche beim Zerreiben ein 
vollkommen weisses Pulver liefern. Die neue Verbindung unterscheidet sich schon in ihrer Krystallform und durch ihre geringere Löslichkeit in Chloroform von der unten zu beschreibenden Phenylchlorjodpropionsäure-Zimmtsäure. Im Capillarrohre erhitzt, beginnt sie bei $100^{\circ}$ roth zu werden und bei $122-123^{\circ}$ schmilzt sie unter Gasentwicklung zu einer tief dunklen Flüssigkeit.

Die Halogenbestimmungen ergaben folgende Resultate:

I. $0,1710 \mathrm{~g}$ gaben $0,2084 \mathrm{AgCl}+\mathrm{AgJ}$.

II. $0,1752 \mathrm{~g} \quad " \quad 0,2144 \mathrm{AgCl}+\mathrm{AgJ}$.

III. $0,2362 \mathrm{~g} \quad, \quad 0,2886 \mathrm{AgCl}+$ AgJ.

IV. $0,2494 \mathrm{~g}, \quad 0,3034 \mathrm{AgCl}+\mathrm{AgJ}$.

\begin{tabular}{cccccc} 
& Berechnet füir & \multicolumn{4}{c}{ Gefunden } \\
\cline { 3 - 6 } & $\mathrm{C}_{9} \mathrm{H}_{\mathrm{B}} \mathrm{ClJO}_{\mathbf{z}}$ & I. & II. & III. & IV. \\
$\mathrm{Cl}$ & 11,43 & $\mathbf{1 1 , 4 3}$ & 11,47 & 11,44 & 11,38 \\
$\mathrm{~J}$ & $\mathbf{4 0 , 9 0}$ & $\mathbf{4 0 , 8 8}$ & $\mathbf{4 1 , 0 6}$ & $\mathbf{4 1 , 0 2}$ & $\mathbf{4 0 , 8 4}$
\end{tabular}

Diese Zahlen stimmen genau auf Phenylchlorjodpropionsäure, die Kohlenstoffbestimmungen gaben etwas zu hohe Werthe:

I. $0,301 \mathrm{~g}$ gaben $0,3910 \mathrm{CO}_{2}$ und $0,0770 \mathrm{H}_{2} \mathrm{O}$.

II. $0,3013 \mathrm{~g} \quad, \quad 0,3920 \mathrm{CO}_{\mathrm{z}}, \quad 0,0724 \mathrm{H}_{\mathrm{g}} \mathrm{O}$.

III. $0,3028 \mathrm{~g} \quad, \quad 0,3920 \mathrm{CO}_{3} \quad, \quad 0,0720 \mathrm{H}_{2} \mathrm{O}$.

\begin{tabular}{ccrrr} 
& Berechnet für & \multicolumn{3}{c}{ Gefunden } \\
\cline { 3 - 5 } C & $\mathrm{C}_{8} \mathrm{H}_{8} \mathrm{ClJO}_{9}$ & I. & II. & III. \\
$\mathrm{H}$ & 34,78 & 35,42 & 35,47 & 35,30 \\
& 2.57 & 2,84 & 2,66 & 2,64
\end{tabular}

Trotzdem ist nicht daran zu zweifeln, dass die untersuchte Verbindung Phenylchlorjodpropionsäure ist. In Chloroform und Benzol ist die Säure in der Wärme leicht, in Ligroïn sehr schwer löslich, deshalb wird eine Lösung in den beiden erstgenannten Flüssigkeiten durch Ligroïn krystallinisch gefällt. Auch in Alkohol ist sie besonders beim Erwärmen leicht löslich, die Lősung färbt sich aber nach kurzer Zeit braun von freigewordenem Jod.

Von Wasser wird die trockne Säure sehr schwer benetzt, bringt man sie mit der zehnfachen Menge Wasser von gewobnlicher Temperatur zusammen, so schwimmt sic anfangs anf dem 
Wasser, aber nach 24 Stunden ist sie in ein schweres Krystallpulver verwandelt, das auf dem Boden liegt, es besteht aus Phenyljodmilchsäure, die neben Salzäure entstanden ist. Trägt man die gepulverte Säure in die zehnfache Menge auf $70^{\circ}$ erhitztes Wasser, so entsteht sofort Phenyljodmilchsäure, welche bei raschem Abkühlen herauskrystallisirt.

Ebenso wird die Phenylchlorjodpropionsäure beim Behandeln mit einer wässrigen Lösung von einem Mol.-Gew. Kaliumhydroxyd oder von 1/2 Mol.-Gew. Soda sofort in Phenyljodmilchsäure übergeführt.

$1 \mathrm{~g}$ Phenylchlorjodpropionsäure wurde mit $0,1707 \mathrm{~g}$ Natriumcarbonat in $10 \mathrm{ccm}$ Wasser gelöst zusammengebracht, dann die Flüssigkeit mit Aether ausgeschüttelt. Die ätherische Lösung hinterliess $0,9 \mathrm{~g}$ Phenyljodmilehsäure statt 0,94 ber.

Durch eine wässrige Jodkaliumlösung wird sie unter Abscheidung von Jod und Bildung von Chlorkalium zu Zimmtsäure reducirt.

$0,2965 \mathrm{~g}$ Phenylchlorjodpropionsäure wurden bei gewöhnlicher Temperatur mit einer wässrigen Lösung von überschüssigem Jodkalium behandelt und das ausgeschiedene Jod mit thioschwefels. Natron bestimmt (1 cem $\mathrm{S}_{2} \mathrm{O}_{3} \mathrm{Na}_{2}=0,0439$ Jod). Verbraucht $5,5 \mathrm{cem}=$ $0,2415 \mathrm{~J}=0,1545 \mathrm{ClJ}=52,11 \mathrm{pC}$. ClJ, berechnet $52,33 \mathrm{pC}$.

Da, wie ich später zeigen werde, die aus der Phenylchlorjodpropionsäure entstehende Phenyljodmilchsäure das Jod in der $\alpha$-, das Hydroxyl in der $\beta$-Stellung enthält, so muss die Phenylchlorjodpropionsänre. Phenyl- $\beta$-chlor- $\alpha$-jodpropionsäure sein und demgemäss durch folgende Formel ausgedrückt werden:

$$
\mathrm{C}_{\mathbf{0}} \mathrm{H}_{5}-\mathrm{CHCl}-\mathrm{CHJ}-\mathrm{COOH} \text {. }
$$

Phenylchlorjodpropionsäure-Methyl- und -Aethylester.

Wenn man Zimmtsäuremethylester mit etwas überschüssiger, etwa 10 procentiger wässriger Chlorjodsalzsäurelösung schüttelt, so bildet sich eine zusammengeballte Masse, die man zweckmässig möglichst fein mit der Flüssigkeit verreibt. Nach einigen Stunden wird der Niederschlag von der Flüssigkeit getrennt, mit Wasser ausgewaschen und uber Schwefelsäure getrocknet. 
Beim Umkrystallisiren aus Ligroïn erhält man farblose, derbe Krystalle, welche am Lichte braun werden und bei $97-98^{\circ}$ unter Röthung schmelzen, auch in Aether, Benzol, Chloroform leicht löslich sind. Die Lösung in Alkohol färbt sich sehr bald braun.

$0,1514 \mathrm{~g}$ gaben $0,1775 \mathrm{AgCl}+\mathrm{AgJ}$.

Berechnet für $\mathrm{C}_{6} \mathrm{H}_{5}$-CHCl-CHJ-COOCH

Cl

$\mathrm{J}$
10,93

39,14
Gefunden

11,04

39,22

Der Aethylester wurde in derselben Weise dargestellt; aus Ligroï ebenfalls derbe, farblose, am Lichte braun werdende Krystalle, die bei $69-70^{\circ}$ unter Röthung schmelzen. Gegen Lösungsmittel verhält er sich dem Methylester ähnlich.

$0,1502 \mathrm{~g}$ gaben $0,1694 \mathrm{AgCl}+\mathbf{A g J}$.

Berechnet für

Gefunden

$\mathrm{C}_{6} \mathrm{H}_{5}$-CHCl-CHJ-COOC $\mathrm{H}_{5}$

$\begin{array}{lll}\text { Cl } & 10,49 & 10,55 \\ J & 37,52 & 37,81\end{array}$

Aus der Phenylchlorjodpropionsäure entsteht durch eine absolut alkoholische Lösung von einem Mol.-Gew. Kaliumhydroxyd

\section{Phenyl- $\beta$-äthoxy- $\alpha$-jodpropionsäure.}

Zur Darstellung dieser Säure löst man am zweckmässigsten jedesmal nur $2 \mathrm{~g}$ Phenylchlorjodpropionsäure in $5 \mathrm{ccm}$ absolutem Alkohol, setzt eine Auflösung von 0,362 $\mathrm{g}$ Kaliumbydroxyd in $5 \mathrm{ccm}$ absolutem Alkohol hinzu und lässt über Nacht stehen. Es scheidet sich Chlorkalium $a b$ und die Flüssigkeit ist von frei gewordenem Jod braun. Sie wird von dem Chlorkalium durch Absaugen getrennt, vorsichtig mit thioschwefelsaurem Natrium entfärbt und mit Wasser gefällt. Dabei wird eine krystallinische und eine ölige, bald erstarrende Ausscheidung erhalten. Man bringt dieselbe aufs Filter, wäscht sie mit Wasser aus und löst sie in Ammoniak. Die etwas ölig getrübte Flüssigkeit wird durch ein nasses Filter gegossen und mit sehr 
verdunnter Schwefelsäure fractionirt gefallt. Der zuerst entstehende flockig-krystallinische Niederschlag wird getrennt, sobald die einfallende Schwefelsäure eine milchige Trübung hervorbringt. Setzt man jetzt mehr Schwefelsäure hinzu, so scheidet sich nach kurzer Zeit die neue Säure in glänzenden Krystallen ab.

Durch Umkrystallisiren aus heissem Wasser erhält man centimeterlange Nadeln, welche ein Molekül Krystallwasser enthalten, das sie über Schwefelsäure abgeben.

$1,066 \mathrm{~g}$ verloren in 50 Stunden $0,0584=5,47 \mathrm{pC}$. Wasser, für 1 Mol. berechnet $5,32 \mathrm{pC}$.

$0,150 \mathrm{~g}$ getrocknet, gaben 0,1097 AgJ.

$0,300 \mathrm{~g} \quad " \quad$ " $0,451 \mathrm{CO}_{2}$ und $0,1086 \mathrm{H}_{2} \mathrm{O}$.

Berechnet für Gefunden $\mathrm{C}_{\mathbf{1 1}} \mathrm{H}_{13} \mathrm{JO}_{\mathbf{8}}$

$\begin{array}{lrr}\mathrm{C} & 41,25 & 41,00 \\ \mathrm{H} & 4,06 & 4,02 \\ \mathrm{~J} & 39,69 & 39,52\end{array}$

Die Säure schmilzt bei $138-139^{\circ}$ unter schwacher Röthung. In heissem Wasser schmilzt sie und löst sich schwer darin auf, in kaltem ist sie kaum löslich, leicht löst sie sich in Alkohol, Benzol, Chloroform, Aether, Eisessig, auch heisses Ligroïn löst sie.

Phenyl- $\beta$-methoxy- $\alpha$-jodpropionsäure wird in gleicher Weise erhalten, wie die Aethoxysäure, wenn man statt Aethylalkohol absoluten Methylalkohol anwendet.

Aus heissem Wasser, unter dem sie nicht schmilat, erhält man sie als wasserfreie Nadeln, welche bei $164-165^{\circ}$ unter Röthung schmelzen.

$0,3017 \mathrm{~g}$ gaben $0,4342 \mathrm{CO}_{2}$ und $0,098 \mathrm{H}_{2} \mathrm{O}$.

$0,1625 \mathrm{~g} \quad " \quad 0,1242 \mathrm{AgJ}$.

$\begin{array}{lcr} & \text { Berechnet fiir } & \text { Gefunden } \\ & \mathrm{C}_{10} \mathrm{H}_{11} \mathrm{JO}_{3} & \\ \mathrm{C} & 39,21 & 39,25 \\ \mathrm{H} & 3,59 & 3,60 \\ \mathrm{~J} & 41,50 & 41,24\end{array}$


Schwer löslich in heissem, kaum in kaltem Wasser, wird sie leicht aufgenommen von Alkohol, Aether, Benzol, Chloroform und Eisessig, sehr schwer von warmem Ligroïn.

NB. Die Aethoxysäure wurde auch noch in folgender Weise gewonnen: $3 \mathrm{~g}$ Zimmtsäure in $7 \mathrm{ccm}$ absolutem Alkohol gelöst wurden mit einer Lösung von $1,14 \mathrm{~g}$ Kaliumhydroxyd in $7 \mathrm{ccm}$ absolutem Alkohol versetzt. Ausscheidung des zimmtsauren Kalium, nun wurde 1/20 Mol.-Gew. Chlorjodsalzsäure in $6 \mathrm{ccm}$ absolutem Alkohol gelöst hinzugefügt, wodurch der Niederschlag in Lösung ging. Dann wurde wieder 1,14 Kaliumhydroxyd in $7 \mathrm{ccm}$ absolutem Alkohol gelöst hinzugemischt: reichliche Ausscheidung von Chlorkalium und Braunfärbung der Flüssigkeit. Die Mischung blieb über Nacht stehen, wurde durch Absaugen vom Chlorkalium befreit, vorsichtig mit thioschwefelsaurem Natron entfärbt und mit Wasser gefällt; der ausgewaschene Niederschlag wurde in Ammoniak gelöst und mit der Lösung wie oben verfahren.

Terhalten der wässrigen Chlorjodsalzsäurelösung gegen Zimmtsäure.

Bringt man bei gewöhnlicher Temperatur eine Lösung von zimmtsaurem Natron mit Chlorjodsalzsäurelösung zusammen, so scheidet sich ein weisser Niederschlag aus, welcher aus reiner Zimmtsäure besteht. Lässt man aber die Zimmtsäure unter öfterem Schñtteln mit der Chlorjodsalzsäurelösung in Bertihrung, so verschwindet sie allmählich und nach $1 \frac{1}{2}$ bis 2 Stunden hat sie sich unter Abscheidung einer kleinen Menge eines braunen Oels aufgelöst. Wenn man jetzt die vom Oel getrennte Flüssigkeit mit einer Lösung von thioschwefelsaurem Natron entfärbt und mit Aether ausschuttelt, so erhält man beim Verdunsten des Aetherextracts farblose Krystalle einer Säure von der Zusammensetzung $\mathrm{C}_{9} \mathrm{H}_{9} \mathrm{JO}_{3}$, welche als Phenyljodhydracrylsäure erkannt wurde.

Das erwähnte braune Oel erstarrt nach einiger Zeit za einem krystallinischen Kuchen, der nach dem Entfärben mit 
Natriumthiosulfat an Aether dieselbe Verbindung abgiebt, wic die wässrige Flüssigkeit.

Wenn man dagegen aus der durch Thiosulfat entfärbten Flüssigkeit das Reactionsproduct durch Abdampfen auf dem Wasserbade gewinnen will, so färbt sich dieselbe durch frei werdendes Jod braun und scheidet, nachdem sie wieder durch Thiosulfat entfärbt ist, beim Erkalten einen krystallinischen Niederschlag von reiner Zimmtsäure ab, welche sich aus der jodhaltigen Säure zurückgebildet hat.

Aus dem Mitgetheilten geht hervor, dass die Zimmtsäure in der Chlorjodsalzsäurelösung nur dann ,ganz unverändert" bleibt, wie Dittmar (Ber. d. deutsch. chem. Ges. 18, 1613) angenommen hat, wenn man sie nur ganz kurze Zeit in Berührung lässt; es ergiebt sich aber ferner noch, dass aus einer Flüssigkeit, in welcher das jodhaltige Reactionsproduct entbalten ist, unter gewissen Umständen Zimmtsäure scheinbar unverändert wieder zum Vorschein kommen kann.

\section{Phenyljodhydracrylsäure.}

Wenn man in der folgenden Weise verfährt, so kann man in kurzer Zeit beliebige Mengen Zimmtsäure fast quantitativ in Phenyljodbydracrylsäure überführen:

Die heisse Lösung von einem Mol.-Gew. zimmtsaurem Natron wird in eine auf $70^{\circ}$ erwärmte fünfprocentige, anderthalb Mol.Gew. Chlorjod enthaltende Chlorjodsalzsäurelösung unter Schütteln eingetragen. Die beim Einfallen der Cinnamatlösung jedesmal entstehende Trübung verschwindet anfangs sogleich wieder, erst gegen Ende des Eintragens bleibt eine geringe Trübung, die sich alsbald auf dem Boden des Gefässes als Oel ansammelt. Giesst man von diesem die noch heisse Flüssigkeit ab und lässt erkalten, so scheidet sich je nach der herrschenden Temperatur bald mehr, bald weniger von der gebildeten jodhaltigen Säure in Krystallen aus. Wird dann dic abgegossene Mutterlauge mit Kochsalz gesättigt, so werden weitere Mengen der Säure krystallinisch ausgeschieden. Den Rest kann man durch Ausschütteln der 
mit Thiosulfat entfärbten Flüssigkeit, sowie des ebenso behandelten Oels, resp. des daraus entstandenen Krystallkuchens mit Aether gewinnen. Die mit Wasser gewaschenen Krystalle werden über Schwefelsäure vollständig getrocknet und aus heissem Benzol umkrystallisirt. Man kann auch die feuchte Säure aus Wasser umkrystallisiren, indem man sie in vorber nahe zum Sieden erhitztes Wasser bis zur Sättigung einträgt und die Lösung abkühlt.

Bei gewöhnlicher Temperatur wurden aus $20 \mathrm{~g}$ Zimmtsäure 29,8 g reine Phenyljodhydracrylsäure gewonnen, die Mutterlaugen wurden auf phenylglycidsaures Natron verarbeitet.

Bei $70^{\circ}$ wurden aus $50 \mathrm{~g}$ Zimmtsäure an Phenyljodhydracrylsäure erhalten:

Durch Krystallisation beim Erkalten der

Reactionsflüssigkeit . . . . . . . . $33 \mathrm{~g}$ Durch Aussalzen . . . . . . . . . . . . . . $45 \mathrm{~g}$

Dureh Ausschütteln mit Aether aus der Flüssigkeit und

dem Oel, das krystallinisch erstarrt war

\begin{tabular}{lr}
$. \quad . \quad 18 \mathrm{~g}$ \\
\hline Znsammen & $96 \mathrm{~g}$ \\
statt $\quad 98,6 \mathrm{~g}$
\end{tabular}

Die über Schwefelsäure getrocknete Säure lieferte bei der Analyse folgende Zahlen:

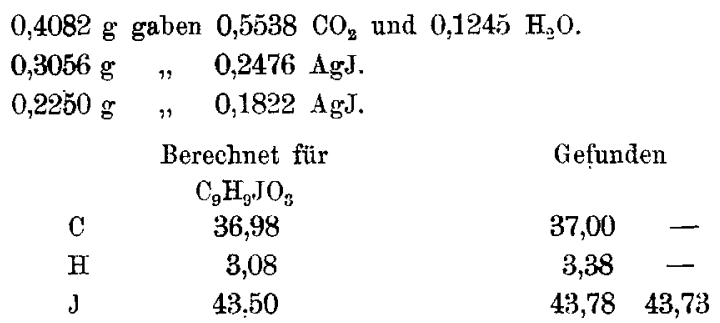

Eigenschaften. Die Säure wird aus Benzol in farblosen, $1 / 2 \mathrm{~cm}$ laugen, flachen Prismen erhalten, welche bei $140-142^{0}$ unter Rothfärbung und Aufschäumen schmelzen. In kaltem Wasser schwer löslich, ein Theil der fein gepulverten Säure löst sich langsam in etwa 200 Theilen Wasser von gewöhnlicher Temperatur, in heissem Wasser ist sie reichlicher löslich. Wenn man die gepulverte Säure mit absolutem Alkohol befeuchtet, 
so reichen 120 Theile kaltes Wasser zur Lösung hin. (Löst man $1 \mathrm{~g}$ der Säure in soviel absolutem Alkohol, dass das Volum der Lösung $3 \mathrm{ccm}$ beträgt, so kann man diese Lösung mit beliebigen Mengen Wasser vermischen, ohne dass Ausscheidung der Säure stattfindet.)

Leicht löslich ist die Säure in Alkohol, Aether, Chloroform, Schwefelkohlenstoff und warmem Benzol und fast unlöslich in Ligroïn.

Wenn man aus Benzol krystallisirte Säure als feines Pulver mit Wasser kalt stehen lässt, so verwandelt sich das Pulver allmählich in grössere Krystalle, welche an der Luft langsam verwittern and über Schwefelsäure oder bei $60^{\circ}$ ein Molekül Wasser verlieren.

I. $0,652 \mathrm{~g}$ verloren über Schwefelsäure 0,0406.

II. $0,507 \mathrm{~g} ", \quad, \quad 0,0290$.

III. $0,8317 \mathrm{~g}, \quad$ bei $60^{\circ} 0,0506$.

\begin{tabular}{cccc} 
& Berechnet für & \multicolumn{3}{c}{ Gefunden } \\
$\mathrm{C}_{9} \mathrm{H}_{9} \mathrm{JO}_{3}+\mathrm{H}_{2} \mathrm{O}$ & $\overbrace{\text { I. }}$ & II. & III. \\
5,80 & 5,22 & 5,71 & 6,08
\end{tabular}

Die Phenyljodhydracrylsäure vermag also, wie die beiden anderen Phenylhalogenhydracrylsäuren, ein Molekül Krystallwasser zu binden.

Wird die Säure mit Wasser auf dem Wasserbade erhitzt, so schmilzt sie zunächst zu einem farblosen Oel und löst sich dann zu einer klaren, farblosen Flüssigkeit auf, die nur sehr schwach den Geruch des Phenyläthylaldehyds zu erkennen giebt. Bei langsamem Abkühlen scheidet sich die Säure zuerst als Oel aus, das langsam erstarrt, bei raschem Abkühlen scheiden sich dieselben wasserfreien Krystalle ab, welche man aus Benzollösung erbält.

Kocht man aber die wässrige Lösung längere Zeit, oder destillirt sie mit Wasserdampf, oder erhitzt sie im zugeschmolzenen Rohre im siedenden Wasserbade, so färbt sie sich braun und scheidet schliesslich krystallinisches Jod aus. Bei der Destillation geht neben Jod Phenyläthylaldehyd, Styrol und etwas 
Zimmtsäure mit dem Wasserdampf über und aus der von Jod befreiten Flüssigkeit in der Retorte krystallisirt beim Erkalten Zimmtsäure aus. Die Bestimmung des Jods und der Zimmtsäure hat ergeben, dass auf ein Mol.-Gew. des ersteren nahezu ein Mol.-Gew, der letzteren gebildet wird.

$3 \mathrm{~g}$ Phenyljodhydracrylsäure mit $30 \mathrm{ccm}$ Wasser sieben Stunden im zugeschmolzenen Rohre bei $100^{\circ}$ erhitzt, ergaben 0,8848 Jod und 0,51 Zimmtsäure statt 0,519, wenn gleiche Mol.-Gew. entstanden wären.

Wahrscheinlich wird von der Phenyljodhydracrylsäure unter Bildung von Phenylglycidsäure resp. deren Zersetzungsproducten Jodwasserstoff abgespalten und dieser wirkt auf weitere Phenyljodhydracrylsäure ein. Beim Erhitzen dieser Säure mit Jodwasserstoff im Wasserbade wurde, wie ein besonderer Versuch zeigte, freies Jod und Zimmtsäure gebildet.

$2 \mathrm{~g}$ der Säure wurden nur kurze Zeit mit $12 \mathrm{pc}$. Jodwasserstofflösung im Wasserbade erhitzt und das freigewordene Jod sowie die Zinmtsäure bestimmt. Gefunden Jod 1,305, Zimmtsäure 0,6. Die Zersetzung war nicht vollständig zu Ende gefuihrt worden, es waren noch 0,7 Phenyljodhydracryls. unverändert geblieben.

Obgleich man nach der relativ grösseren Beständigkeit der Phenyljodmilchsäure beim Behandeln mit Wasser schon den Schluss ziehen konnte, dass in ihr das Jod nicht die $\beta$ - sondern die $\alpha$-Stellung einnehme, so wurde sie doch noch in Phenylmilchsäure verwandelt und diese mit verdünnter Schwefelsäure bei $100^{\circ}$ erhitzt, da nach meinen Versuchen bei dieser Behandlung die Phenyl- $\beta$-hydroxypropionsäure in kurzer Zeit unter Bildung von Zimmtsäure neben etwas Styrol und Kohlendioxyd zersetzt wird, während dagegen die $\alpha$-Hydroxysäure unter den angegebenen Bedingungen ganz unverändert bleibt.

\section{Reduction der Phenylhydroxyjodpropionsäure.}

$10 \mathrm{~g}$ der Säure wurden in der 30 fachen Menge Wasser vertheilt und mit einem Ueberschuss von Natriumamalgam unter Vermeidung von Erwärmung so lange geschüttelt, bis eine constante Wasserstoffentwicklung $\mathbf{z u}$ beobachten war. Die 
filtrirte, mit verdünnter Schwefelsäure neutralisirte Lösung wurde durch Abdampfen auf dem Wasserbade auf ein kleineres Volum gebracht, mit verdünnter Schwefelsäure sauer gemacht und mit Aether ausgeschüttelt. Nach dem Abdestilliren des Aethers schossen lange Prismen an, deren Schmelzpunkt nach dem Umkrystallisiren aus Ligroïn bei $93^{\circ}$ gefunden wurde.

Nachdem diese Saxure mit dem fünffachen Gewichte verdünnter Schwefelsäure (1:3) eine Stunde lang im zugeschmolzenen Rohre in einem siedenden Wasserbade erhitzt worden war, öffnete sich die Spitze des Rohres in der Flamme unter schwachem Druck, die Flüssigkeit enthielt Zimmtsäurekrystalle und roch nach Styrol. Zum Ueberfluss wurde eine andere Portion der Phenylmilchsäure nach Glaser ${ }^{14}$ ) mit concentrirter Salzsäure behandelt und so die bei $126^{\circ}$ schmelzende Phenyl- $\beta$-chlorpropionsäure erhalten, bei deren Behandlung mit kohlensaurem Natron ebenso wie es Fittig und Binder ${ }^{15}$ ) bei der Phenylbrom- und Phenyljodpropionsäure beobachteten, reichliche Mengen von Styrol gebildet wurden. Hiermit war der experimentelle Nachweis geliefert, dass die durch Einwirkung von Chlorjodsalzsäurelösung auf Zimmtsäure entstehende Phenylhydroxyjodpropionsäure das Hydroxyl in der $\beta$ - und das Jod in der $\alpha$-Stellung enthält, dass sie als Phenyljodhydracrylsäure $\mathrm{zu}$ bezeichnen ist.

\section{Verhalten der Phenyljodhydracrylsäure gegen Sodalösung.}

Ein Mol.-Gew. der Säure wurde mit einer Lösung von einem Mol.-Gew. Soda in der fünffachen Menge Wasser unter schwacher Erwärmung so lange geschüttelt, bis alles in Lösung gegangen war. Als nun die Flüssigkeit mit Chlornatrium gesättigt wurde, schied sich das gebildete phenylglycidsaure Natron fast vollständig als krystallinisches Pulver aus und konnte durch mehrmaliges Umkrystallisiren aus Alkohol vollständig rein

14) Diese Annalen 147, 95.

15) Diese Annalen 195, 135 und 137. 
erhalten werden, wie die Natriumbestimmung des über Schwefelsäure getrockneten Salzes beweist.

$0,165 \mathrm{~g}$ gaben $0,0624 \mathrm{Na}_{2} \mathrm{SO}_{4}=12,25 \mathrm{pC}$. Na Berechnet für $\mathrm{C}_{9} \mathrm{H}_{7} \mathrm{O}_{3} \mathrm{Na}$ $=12,36$.

Verhalten der Phenyljodhydracrylsüure gegen Salzsäure.

Nach Glaser ${ }^{16}$ ) wird Phenylbrombydracrylsäure durch concentrirte Chlorwasserstoffsäure in Phenylchlorbrompropionsäure übergeführt, indem das Hydroxyl durch Chlor substituirt wird. Man musste deshalb versuchen, aus der Phenyljodhydracrylsäure in gleicher Weise zur Phenylchlorjodpropionsäure zu gelangen.

Feinst gepulverte Phenyljodhydracrylsäure wurde mit der 15 fachen Gewichtsmenge 38 procentiger Salzsäure übergossen und unter häufigem Umschütteln resp. Umrühren bei gewöhnlicher Temperatur stehen gelassen. Die Flüssigkeit färbt sich bald gelb und das suspendirte Pulver quillt derart auf, dass ein steifer Brei entsteht. Nach etwa fünf Stunden bemerkt man keine weitere Veränderung mehr. Man trennt die Flüssigkeit von dem festen Körper durch Absaugen. Die gelbe Farbe der Flüssigkeit rührt von der bei der Reaction gebildeten Chlorjodsalzsäure her. Den Filterinhalt wäscht man mit kaltem Wasser vollständig aus und bringt ihn über Schwefelsäure und Kalihydrat in den Exsiccator. Der getrocknete feste Körper ist ein aus Nadeln bestehendes krystallinisches Pulver, das unter dem Mikroskop vollständig gleichartig erscheint.

Durch Umkrystallisiren aus heissem Benzol oder Ligroïn bekommt man aus Nadeln bestehende Wärzchen; diese werden aber beim Trocknen oberflächlich gelb, während die ursprünglichen Kryställchen farblos bleiben. Deshalb wurde die frisch bereitete, auf dem Saugfilter mit kaltem Wasser vollständig ausgewaschene, über Schwefelsäure und Kalihydrat getrocknete Verbindung der Untersuchung unterworfen.

16) Diese Annalen 147, 94. 
Die Kryställchen sind in kaltem Wasser unlöslich, leicht in heissem Benzol, schwer in heissem Ligroin löslich. Beim Erhitzen im Capillarrohre werden sie bei $70^{\circ}$ roth, dann immer dunkler, bis bei $110-115^{0}$ unter Flüssigwerden vollständige Zersetzung eintritt.

Die Analysen von drei verschiedenen Darstellungen (I wurde nach 5-, II nach 9-, III nach 24 stündiger Einwirkung verarbeitet) gaben folgende Resultate:

I. $0,3126 \mathrm{~g}$ gaben $0,541 \mathrm{CO}_{2}$ und $0,1077 \mathrm{H}_{2} \mathrm{O}$.

$$
0,2455 \mathrm{~g} \quad, \quad 0,2010 \mathrm{AgCl}+\mathrm{AgJ} \text {. }
$$

II. $0,3120 \mathrm{~g}, \quad 0,539 \mathrm{HO}_{2}$ und $0,1045 \mathrm{H}_{2} \mathrm{O}$.

$0,203 \mathrm{~g} \quad " \quad 0,1674 \mathrm{AgCl}+\mathrm{AgJ}$.

III. $0,3095 \mathrm{~g}, \quad 0,5365 \mathrm{CO}_{2}$ und $0,1045 \mathrm{H}_{2} \mathrm{O}$.

$0,2174 \mathrm{~g} \quad, \quad 0,1794 \mathrm{AgCl}+\mathrm{AgJ}$.

\begin{tabular}{lcrrr} 
& Berechnet für & \multicolumn{3}{c}{ Gefunden } \\
\cline { 3 - 5 } & $\mathrm{C}_{18} \mathrm{H}_{16} \mathrm{ClJO}_{4}$ & I. & \multicolumn{1}{c}{ II. } & \multicolumn{1}{c}{ III. } \\
$\mathrm{C}$ & 47,11 & 47,19 & 47,11 & 47,27 \\
$\mathrm{H}$ & 3,49 & 3,82 & 3,72 & 3,75 \\
$\mathrm{Cl}$ & 7,74 & 7,68 & 7,73 & 7,72 \\
$\mathrm{~J}$ & 27,61 & 27,47 & 27,63 & 27,65
\end{tabular}

Hiernach hat die Verbindung die Zusammensetzung $\mathrm{C}_{18} \mathrm{H}_{16} \mathrm{ClJO}_{4}$. Sie enthält die Bestandtheile von einem Mol. Phenylchlorjodpropionsäure und einem Mol. Zimmtsäure ${ }^{17}$ ).

Beim Erwärmen mit Wasser wird sie in Zimmtsäure, Phenyljodhydracrylsäure und Salzsäure zersetzt.

17) Wenn man die Phenyljodhydracrylsäure statt mit der 15 fachen, mit der 30 fachen Gewichtsmenge 38 procentiger Salzsäure 5 Stunden in Berührung lässt, so erhält man neben der Phenylchlorjodpropionsäure-Zimmtsäure auch freie Zimmtsäure, wie die Analyse eines solchen Gemenges ergiebt:

$0,3157 \mathrm{~g}$ gaben $0,564 \mathrm{CO}_{2}$ und $0,107 \mathrm{H}_{2} \mathrm{O}$.

$0,2206 \mathrm{~g} \quad, \quad 0,1709 \mathrm{AgCl}+\mathrm{AgJ}$.

$\begin{array}{lc} & \text { Gefunden } \\ \mathrm{C} & 48,72 \\ \mathrm{H} & 3,76 \\ \mathrm{Cl} & 7,32 \\ \mathrm{~J} & 25,89\end{array}$


$2 \mathrm{~g}$ der Verbindung wurden in einer Retorte in heissem Wasser gelöst und mit Wasserdampf destillirt. Im Destillat: 0,1016 Jod, entsprechend 0,23 zersetzter Jodhydracrylsäure, in der Retorte 0,65 Zimmtsäure und 1,03 Phenyljodhydracrylsäure. Bei glatter Zersetzung mïsste erhalten werden: 0,646 7immtsäure und 1,27 Phenyljodhydracrylsäure.

Durch Natriumamalgam und Wasser wird daraus Phenylpropionsänre erzeugt; durch eine kalte, wässrige Lösung von Jodkalium wird sie unter Abscheidung von Jod in Zimmtsäure verwandelt.

$1 \mathrm{~g}$ mit $0,8 \mathrm{~g} \mathrm{KJ}$ in Wasser gelöst behandelt gab 0,631 Zimmtsänre vom Schmelzp. $133^{\circ}$ stait 0,646 .

Es ist daher nicht sehr wahrscheinlich, dass eine Verkettung von Kohlenstoffaffinitäten stattgefunden hat. Wie ich mir die Vereinignng der beiden Säuren denke, habe ich oben Seite 266 angegeben. Man kann sie als Phenylchlorjodpropionsäure-Zimmtsäure bezeichnen.

Diese Verbindung wird nicht erzeugt, wenn man verdünntere Salzsäure auf die Phenyljodhydracrylsäure einwirken lässt. Bringt man diese als feinstes Pulver mit der 15 fachen Menge 25 procentiger Salsäure zusammen und lässt nnter beständigem Rühren fünf Stunden einwirken, so fürbt sich die Flüssigkeit gelb von frei gewordener Chlorjodsalzsäure und die feste Masse schwillt zu grösserem Volum an. Wird die Flüssigkeit abgesaugt, so lässt sich der Filterinhalt sofort als reine Zimmtsäure identificiren. Nach dem Auswaschen und Trocknen betrug deren Gewicht 4,3 g. Dem Filtrat konnten nach Zerstörung des Chlorjods mit Thiosulfat noch 0,13 unveränderte Phenyljodhydracrylsäure entzogen werden. Durch die verdünntere Salzsäure wurden also $98 \mathrm{pC}$. der in Reaction gesetzten Säure in Zimmtsäure verwandelt.

Die Untersuchung der Phenylchlorjodpropionsäure und Derivate derselben habe ich in Gemeinschaft mit Herrn Dr. J. Rosenhek begonnen und dann zum Theil mit Unterstützung des Herrn Dr. Kautz weiter geführt. Ich spreche diesen Herren für Mitwirkung und Hülfe meinen besten Dank aus. 
Ich hatte eigentlich die Absicht, der Vollständigkeit wegen auch Phenylbromjodpropionsäure darzustellen und zu untersuchen, bin aber̀ nur dazu gekommen, mich zu überzeugen, dass durch Einwirkung einer wässrigen Lösung von Bromjodsalzsäure auf Zimmtsäure dieselbe Phenyljodhydracrylsäure entsteht, welche mit Chlorjodsalzsäure erhalten wird. Es unterliegt demnach wohl keinem Zweifel, dass bei Anwendung von trockner Bromjodsalzsäure Phenyl- $\beta$-brom- $\alpha$-jodpropionsäure zu gewinnen ist.

Auch wollte ich noch versuchen, Chlorbrom an Zimmtsäure anzulagern, weil ich mir dachte, es könnten sich als Chlorbromadditionsproducte der Zimmtsäure die zwei isomeren Phenylchlorbrompropionsäuren neben einander bilden, welche durch Einwirkung von Bromwasserstoff auf Phenylchlorhydracrylsäure und von Chlorwasserstoff auf Phenylbromhydracrylsäure erhalten werden.

Bei einem Versuche, den ich in wässriger Lösung bei $48^{\circ}$ mit Chlorbromsalzsäure ausfüturte, bekam ich aber neben Bromstyrol und Phenylbrombydracrylsäure von $13 \mathrm{~g}$ angewendeter Zimmtsäure $6 \mathrm{~g}$ eines bei $179^{\circ}$ schmelzenden Dihalogenürs, welches mit Wasserdampf destillirt, auch Phenylbromhydracrylsäure und Bromstyrol, mit Soda Bromstyrol lieferte, so dass sich bei der Addition nur die eine Phenylchlorbrompropionsaure zu bilden scheint, welche das negativere Chlor in der $\beta$-, das weniger negative Brom in der $\alpha$-Stellung enthält. Leider bin ich nicht mehr dazu gekommen, den Versuch zu wiederholen und ein gründliches Studium der Reaction unter verschiedenen Versuchsbedingungen vorzunehmen. 